\title{
Phenomenological two-gap model for the specific heat of $\mathrm{MgB}_{2}$
}

\author{
F. Bouquet ${ }^{1,2}$, Y. WAnG ${ }^{1}$, R. A. Fisher ${ }^{2}$, D. G. Hinks ${ }^{3}$, J. D. Jorgensen ${ }^{3}$, \\ A. JunOD ${ }^{1}$, and N. E. Phillips ${ }^{2}$ \\ 1 Département de Physique de la Matière Condensée, Université de Genève - CH-1211 \\ Genève 4 (Switzerland) \\ 2 Lawrence Berkeley National Laboratory and Department of Chemistry, University of \\ California - Berkeley, CA 94720 (USA) \\ 3 Materials Science Division, Argonne National Laboratory - Argonne, IL 60439 (USA)
}

PACS. 74.25.Bt - Thermodynamic properties.

PACS. 74.20.De - Phenomenological theories.

PACS. 74.60.-w - Type-II superconductivity.

\begin{abstract}
We show that the specific heat of the superconductor $\mathrm{MgB}_{2}$ in zero field, for which significant non-BCS features have been reported, can be fitted, essentially within experimental error, over the entire range of temperature to $T_{\mathrm{c}}$ by a phenomenological two-gap model. The resulting gap parameters agree with previous determinations from band-structure calculations, and from various spectroscopic experiments. The determination from specific heat, a bulk property, shows that the presence of two superconducting gaps in $\mathrm{MgB}_{2}$ is a volume effect.
\end{abstract}

The discovery of superconductivity in $\mathrm{MgB}_{2}$ [1] raised the questions of its nature and the origin of its relatively high transition temperature $T_{\mathrm{c}} \sim 40 \mathrm{~K}$. Specific heat $(C)$ is a powerful tool to aid in answering these questions and, more generally, to provide information on the thermodynamics of the transition. Several groups have reported such measurements on $\mathrm{MgB}_{2}$ [2-10]. It is now established that $C$ significantly deviates from the standard BCS behaviour. First, a large excess in $C$ is observed in the vicinity of $T_{\mathrm{c}} / 4$ [2-6]. Second, an exponential fit of $C(T)$ in the region $T \ll T_{\mathrm{c}}$ indicates a gap ratio $2 \Delta_{0} / \mathrm{k}_{\mathrm{B}} T_{\mathrm{c}}$ only onequarter to one-third of the isotropic BCS value $[3,4,6]$. This excess was interpreted as a possible sign of a second superconducting gap, whose existence is predicted by band-structure calculations [11-13]. The specific heat near $T_{\mathrm{c}}$ is puzzling also with the jump $\Delta C$ at $T_{\mathrm{c}}$ consistently smaller than the BCS weak-coupling lower bound. In this Letter, we present an empirical two-gap model that fits the experimental data over the whole range of temperature to $T_{\mathrm{c}}$. This model resolves the apparent contradiction between different analyses of the specific heat, and relevant parameters show good agreement with determinations based on independent experiments.

We focus on two sets of specific-heat data obtained independently in two different laboratories. Experimental methods and results have been described elsewhere $[2,3,5,6]$. The unusual excess specific heat at $\sim T_{\mathrm{c}} / 4$, which denotes the presence of excitations within the

(c) EDP Sciences 
main gap, is a consistent feature that is common to different samples and different techniques. These measurements also give similar values for the normal-state contribution, with a coefficient of the linear term $\gamma_{\mathrm{n}} \sim 2.65(15) \mathrm{mJ} \mathrm{mol}^{-1} \mathrm{~K}^{-2}$, and satisfy the criterion of the normaland superconducting-state entropy being equal at $T_{\mathrm{c}}$. However, detailed results, such as the height and the width of the jump $\Delta C$ at $T_{\mathrm{c}}$, are sample-dependent. The sample of Ref. [3] was a powder of isotopically pure $\mathrm{Mg}^{11} \mathrm{~B}_{2}$ embedded in GE7031 varnish, whereas the sample of Ref. [2] was a sintered commercial powder. A third sample prepared from $\mathrm{Mg}$ and B by high-pressure techniques gave similar results [5]. The electronic part of the specific heat was determined by subtraction of the normal-state data, obtained either at fields of 14 or $16 \mathrm{~T}$ in Ref. [2], or with a short extrapolation of the $9 \mathrm{~T}$ data in Ref. [3]. We refer to the original articles for details.

Although the low- $T$ behaviour of the specific heat data in the earlier studies [2,3] definitely pointed to the presence of excitations with a characteristic energy smaller than the BCS gap $\Delta_{B C S}=3.53 \mathrm{k}_{\mathrm{B}} T_{\mathrm{c}}$, it was not clear whether this was due to a continuous, but extreme, distribution of the gap resulting from anisotropy, or two discrete values of the gap closing at the same temperature $T_{\mathrm{c}}$, with possible anomalous temperature dependence at some intermediate temperature. Furthermore, it was not clear whether these models could account for the specific heat over the whole range of temperature to $T_{\mathrm{c}}$. We present here a simple empirical model, based on the existence of two discrete gaps $\Delta_{1}$ and $\Delta_{2}$ at $T=0$, both closing at $T_{\mathrm{c}}$. In order to calculate their respective contributions, we first consider the case of a single gap $\Delta_{0}$, following the method developed by Padamsee et al., and generally referred to as the $\alpha$-model [14]. The ratio $2 \Delta_{0} / \mathrm{k}_{\mathrm{B}} T_{\mathrm{c}}$ (3.53 in the BCS theory) is not fixed, but is considered to be a fitting variable. The temperature dependence is taken to be the same as in the BCS theory, i.e. $\Delta(t)=\Delta_{0} \delta(t)$, where $\delta(t)$ is the normalised BCS gap at the reduced temperature $t=T / T_{\mathrm{c}}$ as tabulated by Mühlschlegel [15]. The thermodynamic properties, entropy $(S)$ and $C$, can be calculated as appropriate for a system of independent fermion quasiparticles:

$$
\frac{S}{\gamma_{\mathrm{n}} T_{\mathrm{c}}}=-\frac{6}{\pi^{2}} \frac{\Delta_{0}}{\mathrm{k}_{\mathrm{B}} T_{\mathrm{c}}} \int_{0}^{\infty}[f \ln f+(1-f) \ln (1-f)] \mathrm{d} y, \quad \frac{C}{\gamma_{\mathrm{n}} T_{\mathrm{c}}}=t \frac{\mathrm{d}\left(S / \gamma_{\mathrm{n}} T_{\mathrm{c}}\right)}{\mathrm{d} t}
$$

where $f=[\exp (\beta E)+1]^{-1}$ and $\beta=\left(\mathrm{k}_{\mathrm{B}} T\right)^{-1}$. The energy of the quasiparticles is given by $E=\left[\varepsilon^{2}+\Delta^{2}(t)\right]^{0.5}$, where $\varepsilon$ is the energy of the normal electrons relative to the Fermi surface. The integration variable is $y=\varepsilon / \Delta_{0}$.

The fit of experimental data for $\mathrm{MgB}_{2}$ leads to very low values of $2 \Delta_{0} / \mathrm{k}_{\mathrm{B}} T_{\mathrm{c}}$ for one of the gaps, substantially less than 3.53 (see below). The $\alpha$-model was devised for simulation of strong-coupling effects [14], and has usually been applied to strong-coupling superconductors, leading to values $>3.53$. In that case, the temperature at which the gap closes is lowered relative to the normal BCS closing temperature by retardation effects. Since the BCS ratio, $2 \Delta_{0} / \mathrm{k}_{\mathrm{B}} T_{\mathrm{c}}=3.53$, is the weak-coupling lower limit, smaller values can have no physical meaning as measures of the strength of the coupling. (However, anisotropy, both as theoretically studied [16] and experimentally observed [17], does lead to values $<3.5$.) In the present case, as applied to a two-gap superconductor, a small value of $2 \Delta_{0} / \mathrm{k}_{\mathrm{B}} T_{\mathrm{c}}$ has no bearing on the strength of the coupling, but means only that the temperature at which the small gap closes is raised relative to the normal BCS closing temperature by coupling to a larger gap.

Figure 1 shows the calculated $C / t \gamma_{\mathrm{n}} T_{\mathrm{c}}$ for $1 \leq 2 \Delta_{0} / \mathrm{k}_{\mathrm{B}} T_{\mathrm{c}} \leq 5$. We checked the numerical results by comparing the data for $2 \Delta_{0} / \mathrm{k}_{\mathrm{B}} T_{\mathrm{c}}=3.53$ with Mühlschlegel's tables [15], and by verifying that the entropy at $T_{\mathrm{c}}$ is equal to that of the normal state. The curves for $2 \Delta_{0} / \mathrm{k}_{\mathrm{B}} T_{\mathrm{c}} \geq 3.5$ are similar to those reported in Ref. [14]. The unusual shape of the curves for low values of $2 \Delta_{0} / \mathrm{k}_{\mathrm{B}} T_{\mathrm{c}}$ may be understood by considering two characteristic temperatures, 


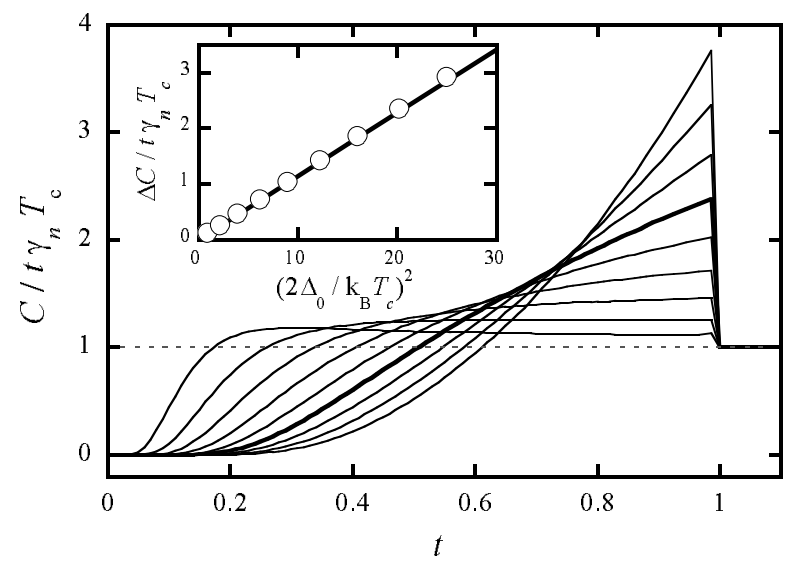

Fig. $1-C / t \gamma_{\mathrm{n}} T_{\mathrm{c}}$ vs. $t$ according to the $\alpha$-model for $2 \Delta_{0} / \mathrm{k}_{\mathrm{B}} T_{\mathrm{c}}=1,1.5,2,2.5,3,3.5(\mathrm{BCS}), 4,4.5,5$. Inset: specific-heat jump at $T_{\mathrm{c}}$ vs. $\left(2 \Delta_{0} / \mathrm{k}_{\mathrm{B}} T_{\mathrm{c}}\right)^{2}$.

$T_{\Delta}=\Delta_{0} /\left(1.76 \mathrm{k}_{\mathrm{B}}\right)$, and $T_{\mathrm{c}}$, which are equal in the BCS limit, but which are independent in the present model:

- For $T \ll T_{\Delta}$, the thermal energy is too small for many quasiparticles to be excited across the gap. Only the tail of the statistical distribution contributes, so that the electronic specific heat follows an exponential behaviour approximately, similar to that of a semiconductor.

- Above $T \approx T_{\Delta}<T_{\mathrm{c}}$, the temperature is high enough to excite most of the quasiparticles across the gap. The specific heat approaches that of the normal state, although the system is still superconducting.

- At $T=T_{\mathrm{c}}$, the gap closes. If $T_{\mathrm{c}} \gg T_{\Delta}$, i.e. if the gap is small compared to the thermal energy at $T_{\mathrm{c}}$, only a small change occurs in the number of excited quasiparticles. The BCS ground state is essentially empty. As a consequence, the specific-heat jump is small.

The smaller the gap, the closer the $C / t \gamma_{\mathrm{n}} T_{\mathrm{c}}$ curve approaches the normal-state line, and the smaller the $\Delta C$ at the transition. We verify numerically the relation between the gap and the jump, $\Delta C=\mathrm{k}_{\mathrm{B}} N(0) /\left(\mathrm{k}_{\mathrm{B}} T_{\mathrm{c}}\right)^{2}\left(\mathrm{~d} \Delta^{2} / \mathrm{d} \beta\right) \propto \Delta_{0}^{2}$ (inset of Fig. 1) [18]. This quadratic dependence holds only because the variation of the normalised gap with $t$ is common to all curves.

In a two-band, two-gap model, the total specific heat can be considered as the sum of the contributions of each band calculated independently according to eq. (1) if interband transitions due to scattering by impurities or phonons can be neglected. Each band is characterised by a partial Sommerfeld constant $\gamma_{i}$, with $\gamma_{1}+\gamma_{2}=\gamma_{\mathrm{n}}$. $C$ data are fitted with three free parameters, the gap widths $\Delta_{1}$ and $\Delta_{2}$, and the relative weights $\gamma_{1} / \gamma_{\mathrm{n}} \equiv x$ and $\gamma_{2} / \gamma_{\mathrm{n}} \equiv 1-x$. Figure 2 shows the data (circles) and the fit (thick line), compared to the BCS specific heat (thin line). Insets show the gap functions, and the various contributions to the total electronic specific heat. The latter curves show evidence of weak correlation between the fitting parameters; the low-temperature excess is related to $\Delta_{2}$, whereas the jump at $T_{\mathrm{c}}$ is due essentially to the $\Delta_{1}$ component. Numerical results are given in Table I. 

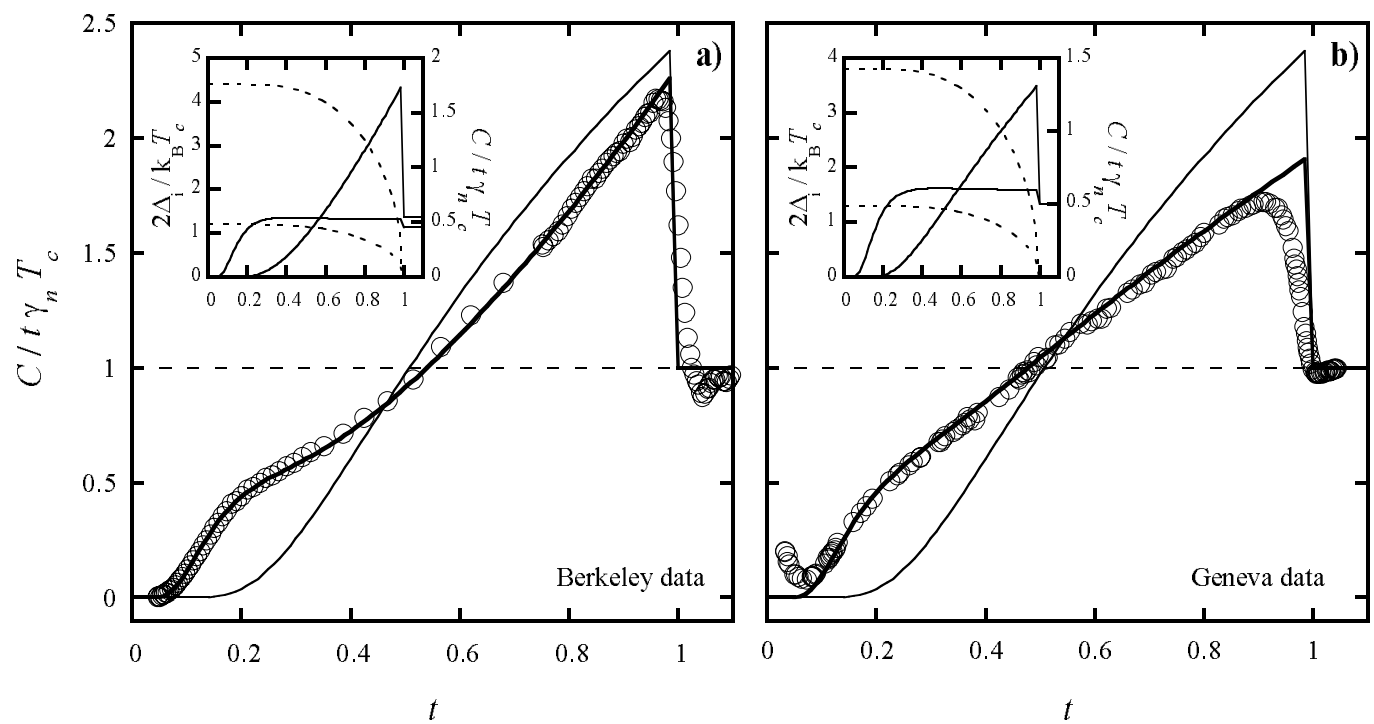

Fig. 2 - BCS normalized specific heat (thin line), experimental data (o), and two-gap fits (thick lines), versus the reduced temperature $t$. (a) data from Ref. [3]; (b) data from Ref. [2]. Insets: gaps $2 \Delta_{1} / \mathrm{k}_{\mathrm{B}} T_{\mathrm{c}}$ and $2 \Delta_{2} / \mathrm{k}_{\mathrm{B}} T_{\mathrm{c}}$ versus $t$ (dotted lines), and partial specific heat of both bands (full lines). Parameters obtained from the fits are given in Table I.

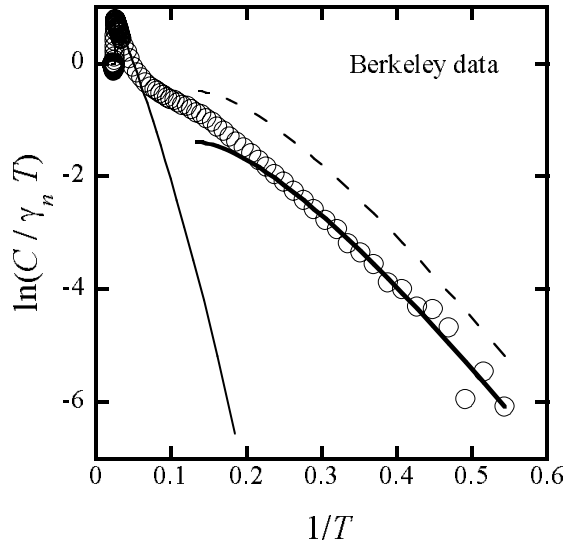

Fig. 3

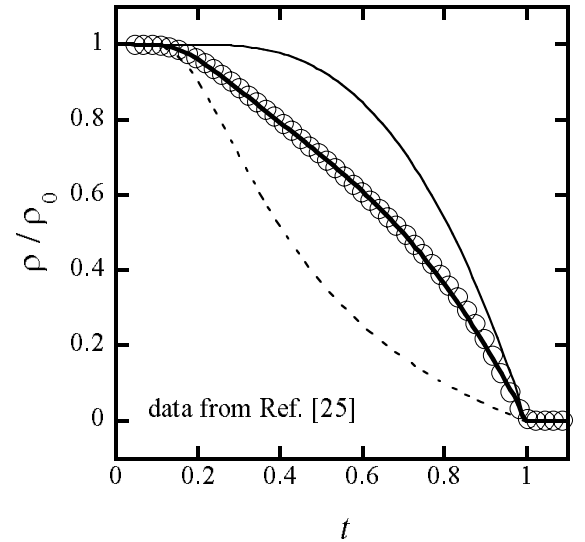

Fig. 4

Fig. 3 - Semi-logarithmic plot of the electronic specific heat versus $1 / T$. Dashed line: asymptotic curve, eq. (2) with $\gamma=\gamma_{\mathrm{n}}$; thick line: eq. (2) with $\gamma=0.4 \gamma_{\mathrm{n}}$ (see text); thin line: standard BCS curve, also shown in Fig. 2; (०), data from Ref. [3].

Fig. 4 - Superfluid fraction versus reduced temperature. Thin line: contribution of $\Delta_{1}$; dotted line: contribution of $\Delta_{2}$; thick line: full two-gap fit; (o): data obtained from measurements of the penetration depth presented in Ref. [25]. Fitted parameters are given in Table I. 
TABLE I - Gap ratios $2 \Delta_{1} / \mathrm{k}_{\mathrm{B}} T_{\mathrm{c}}, 2 \Delta_{2} / \mathrm{k}_{\mathrm{B}} T_{\mathrm{c}}$ and weights $x$ as determined by the two-gap model (lines 1-4) and by different techniques (lines 5-10).

\begin{tabular}{lcccc} 
Ref. & Technique & $2 \Delta_{1} / \mathrm{k}_{\mathrm{B}} T_{\mathrm{c}}$ & $2 \Delta_{2} / \mathrm{k}_{\mathrm{B}} T_{\mathrm{c}}$ & $x:(1-x)$ \\
\hline$[3]$ & specific heat & 4.4 & 1.2 & $55 \%: 45 \%$ \\
{$[2]$} & specific heat & 3.8 & 1.3 & $50 \%: 50 \%$ \\
{$[5]$} & specific heat & 3.9 & 1.3 & $50 \%: 50 \%$ \\
{$[25]$} & penetration depth & 4.6 & 1.6 & $60 \%: 40 \%$ \\
{$[19]$} & Raman & 3.7 & 1.6 & \\
{$[20]$} & photoemission & 3.6 & 1.1 & \\
{$[21]$} & tunneling & 4.5 & 1.9 & \\
{$[22]$} & point-contact spectroscopy & 4.1 & 1.7 & $53 \%: 47 \%$ \\
{$[23]$} & point-contact spectroscopy & 4.2 & 1.0 &
\end{tabular}

In spite of its limitations, this empirical model fits the measured specific heat well over the whole range of $T$ to $T_{\mathrm{c}}$. The sample dependence of the results is reasonably low, and may reflect metallurgical differences. The larger value of $\Delta_{1}$ for the sample of Fig. 2a (isotopically pure $\mathrm{Mg}^{11} \mathrm{~B}_{2}$ powder [3]) reflects a sharper jump and a steeper slope just below $T_{\mathrm{c}}$ compared to the sample of Fig. $2 \mathrm{~b}\left(\mathrm{MgB}_{2}\right.$ sinter [2]). On average, $2 \Delta_{1} / \mathrm{k}_{\mathrm{B}} T_{\mathrm{c}} \sim 4.0$ and $2 \Delta_{2} / \mathrm{k}_{\mathrm{B}} T_{\mathrm{c}} \sim 1.2$, with approximately equal weights.

Moreover, the fitted parameters are qualitatively and quantitatively comparable with independent determinations from other sources. They are consistent with band-structure calculations [13] and spectroscopic measurements [19-23], which report the presence of two gaps, the smaller gap having approximately one-third the BCS value and the larger gap being slightly greater than the BCS value (Table I). We emphasise that $C$, a thermodynamic property, probes the whole volume, whereas spectroscopic measurements are more sensitive to surface conditions.

The relative weights $(1: 1$, i.e. $x \sim 0.5)$ are consistent with the calculations of Ref. [13]. Liu et al. attribute the larger gap $\Delta_{1}$ to particular 2D sheets of the Fermi surface, whereas the smaller gap $\Delta_{2}$ is associated with $3 \mathrm{D}$ sheets. Using partial densities of states and de Haas-van Alphen mass renormalizations, the weight of the smaller gap is evaluated as $x \sim 0.47$, and $1-x \sim 0.53$ for the larger one. The agreement with the two-gap model fits is remarkable.

The present two-gap model reconciles the apparently conflicting results of Ref. [9] and [3,4]. By fitting their specific heat data close to $T_{\mathrm{c}}$, Kremer et al. [9] concluded that their data was consistent with a medium- to strong-coupling $2 \Delta_{0} / \mathrm{k}_{\mathrm{B}} T_{\mathrm{c}} \sim 4.2$. However, the fitted value of $\gamma_{\mathrm{n}}$ at $T_{\mathrm{c}}$ was $1.1 \mathrm{~mJ} \mathrm{~mol}{ }^{-1} \mathrm{~K}^{-2}$, less than half of $\gamma_{\mathrm{n}}$ measured in the normal state. Alternatively, Yang et al. [4] and Bouquet et al. [3] fitted the exponential decrease of the low- $T$ data and concluded that $2 \Delta_{0} / \mathrm{k}_{\mathrm{B}} T_{\mathrm{c}} \sim 0.9$. However, the fitted value of $\gamma_{\mathrm{n}}$ at low $T$ was too small also, $0.7 \mathrm{~mJ} \mathrm{~mol}^{-1} \mathrm{~K}^{-2}$ in Ref. [3]. In the framework of the two-gap model, the main contribution just below $T_{\mathrm{c}}$ is that of the larger gap $\Delta_{1}$, with a break in the slope characteristic of mediumto strong-coupling, and an amplitude of $\Delta C$ determined by $\gamma_{1}=x \gamma_{\mathrm{n}} \sim \gamma_{\mathrm{n}} / 2$ (insets of Fig. 2), in qualitative agreement with Kremer's analysis. The main contribution at $T \ll T_{\mathrm{c}}$ is that of the smaller gap $\Delta_{2}$, with the exponential decrease determined by $\Delta_{2}$, and the amplitude by $\gamma_{2}=(1-x) \gamma_{\mathrm{n}} \sim \gamma_{\mathrm{n}} / 2$ (insets of Fig. 2), again in qualitative agreement with the analysis of Ref. [3]. The latter data are presented below in a slightly different approach. Rather than the 
usual empirical interpolation $C \propto \exp \left(-1.44 T_{\mathrm{c}} / T\right)$, we use the low- $T$ asymptotic formula [24]:

$$
\lim _{T \rightarrow 0} \frac{C}{\gamma T}=3.15\left(\frac{\Delta_{0}}{1.76 \mathrm{k}_{\mathrm{B}} T}\right)^{5 / 2} \exp \left(-\frac{\Delta_{0}}{\mathrm{k}_{\mathrm{B}} T}\right) .
$$

In Fig. 3, we plot data in the form $\ln \left(C / \gamma_{\mathrm{n}} T\right)$ versus $1 / T$, together with the limit given by eq. (2). With $2 \Delta_{0} / \mathrm{k}_{\mathrm{B}} T_{\mathrm{c}}=0.9$ and $\gamma=\gamma_{\mathrm{n}}$, eq. (2) overestimates the data, although the slope determined by $\Delta_{0}$ is correct. With $\gamma \cong 0.4 \gamma_{\mathrm{n}}$, the fit is good in the domain where eq. (2) holds.

The same two-gap model can be applied to the superfluid density $\rho$, which is given, for a single gap, by $\rho=1-2 \Delta_{0} / \mathrm{k}_{\mathrm{B}} T \int_{0}^{\infty} f(1-f) \mathrm{d} y$. The penetration depth $\lambda \propto \rho^{-1 / 2}$ is given in Ref. [25] and is plotted in Fig. 4, together with a two-gap fit (thick line) and its components (full and dotted lines). These data are not strictly bulk measurements, but probe the sample to a typical depth of $\lambda \cong 1800 \AA$ [2]. Nevertheless, $\lambda$ is large compared to the typical sampling depth of many spectroscopic experiments, which is on the scale of the coherence length $\xi \cong 50 \AA$ [2]. The fitted parameters $2 \Delta_{i} / \mathrm{k}_{\mathrm{B}} T_{\mathrm{c}}$ and $x$ are consistent with other determinations (Table I).

The empirical $\alpha$-model allows a quantitative comparison to be made between different experiments and theory within a general framework. The results are numerically consistent, and confirm the coexistence of two gaps for the bulk sample. This situation holds the promise of interesting single-crystals properties. Our two-gap model is phenomenological since we postulate the existence of the gaps, without specifying their origin. Any theoretical approach leading to a similar average electronic density of states would be compatible with the present results, so that specific-heat measurements alone cannot settle in favour of any particular microscopic model $[11,13]$.

Some limitations exist. First, the $\alpha$-model assumes a BCS-like $T$-dependence of the gap. However, if the variation of the smaller gap is reasonably smooth, the results should not depend critically on its exact shape, since the main effect on the specific heat occurs below $T_{\Delta}$ where $\Delta_{2}(T)$ is expected to be essentially constant. Self-consistent calculations of $\Delta(T)$ might lead to corrections, and more elaborate simulations are currently under way [26]. Second, we calculate each contribution of the gaps independently and assume that they are additive. Some coupling is present, but within the present model, its sole effect amounts to bringing the natural closing temperature of the smaller gap, i.e. $\approx 10 \mathrm{~K}$, up to $\approx 40 \mathrm{~K}$.

Our two-gap model describes only the zero-field specific heat. As data in $H>0$ suggest a different field dependence for each gap, a theory of the mixed-state specific heat for a two-gap superconductor would be most useful in extracting quantitative information from $C(T, H)$. Indeed, the field dependence of the electronic contribution at low temperature is unusual. The coefficient of the linear term in the mixed-state, $\gamma(H)$, dramatically increases in small fields [2-6], in a quasi-logarithmic way [5], and saturates for fields much below $H_{\mathrm{c} 2}$, in fact near $H_{\mathrm{c} 2} / 2$. Moreover, the characteristic dip in $C / T$ for $0<T<10 \mathrm{~K}$ associated with the gap $\Delta_{2}$ in one of the bands vanishes by $\approx 0.5 \mathrm{~T}$. Qualitatively, these results would seem to indicate that a small field is able to quench the smaller gap, in agreement with spectroscopic measurements [22]. The saturation of $\gamma(H)$ much below $H_{\mathrm{c} 2}$ suggests that a major part of the electrons are either normal or in a gapless state, possibly by virtue of inter-band scattering in the presence of a normal sheet on the Fermi surface. Forthcoming models may have to embody the different dimensionality of the gaps. Interesting developments are expected for the physics of the vortex state for superconductors with such an unusual $k$-dependent gap. 
Stimulating discussions with I. L. Mazin, A. Carrington, V. Kresin, E. Schachinger, S. V. Shulga and S.-L. Drechsler are gratefully acknowledged. This work was supported by the Fonds National Suisse de la Recherche Scientifique and by the US Department of Energy.

\section{REFERENCES}

[1] Nagamatsu J., Nakagawa N., Muranaka T., Zenitani Y. and Akimitsu J., Nature, 410 (2001) 63.

[2] Wang Y., Plackowski T. and Junod A., Physica C, 355 (2001) 179.

[3] Bouquet F., Fisher, R. A., Phillips N. E., Hinks D. G. and Jorgensen J. D., to be published in Phys. Rev. Letters 87, 047001 (2001); preprint cond-mat/0104206.

[4] Yang H. D., Lin J.-Y., Li H. H., Hsu F. H., Liu C. J. and Changqi Jin, cond-mat/0104574.

[5] Junod A., Wang Y., Bouquet F. and Toulemonde P., to appear in Studies of High Temperature Superconductors Vol. 38, Ed. A.V. Narlikar, Nova Science Publishers, Commack, N.Y.; cond-mat/0106394.

[6] Fisher R. A., Bouquet F., Phillips N. E., Hinks D. G. and Jorgensen J. D., to appear in Studies of High Temperature Superconductors Vol. 38, Ed. A.V. Narlikar, Nova Science Publishers, Commack, N.Y.; cond-mat/0107072.

[7] Marcenat C., Pautrat A., Lawrie D. D., Franck J. P. and Zhang G., to be published.

[8] Bud'ko S. L., Lapertot G., Petrovic C., Cunningham C. E., Anderson N. and Canfield P. C., Phys. Rev. Lett., 86 (2001) 1877.

[9] Kremer R. K., Gibson B. J. and Ahn K., cond-mat/0102432v2.

[10] Wälti Ch., Felder E., Degen C., Wigger G., Monnier R., Delley B. and Ott H. R., cond-mat/0102522.

[11] Shulga S. V., Drechsler S.-L., Eschrig H., Rosner H. and Pickett W., condmat/0103154.

[12] Kortus. J., Mazin I. I., Belashchenko K. D., Antropov V. P. and Boyer L. L., Phys. Rev. Letters, 86 (2001) 4656.

[13] LiU A. Y., Mazin I. I. and Kortus J., cond-mat/0103570.

[14] Padamsee H., Neighbor J. E. and Shiffman C. A., J. Low Temp. Phys., 12 (1973) 387.

[15] Mühlschlegel B., Z. Physik, 155 (1959) 313.

[16] Clem J. R., Annals Phys., 40 (1966) 268.

[17] Okamoto H., Taniguti H. and Ishinara Y., Phys. Rev. B, 53 (1996) 384.

[18] Bardeen J., Cooper L. N. and Schrieffer J. R., Phys. Rev., 108 (1957) 1175.

[19] Chen X. K., Konstantinovic M. J., Irwin J. C., Lawrie D. D. and Franck J. P., condmat/0104005v2.

[20] Tsuda S., Yokoya T., Kiss T., Takano Y., Togano K., Kitou H., Ihara H. and Shin S., cond-mat/0104574.

[21] Giubileo F., Roditchev D., Sacks W., Lamy R., Thanh D. X., Klein J., Miraglia S., Fruchart D., Marcus J. and Monod Ph., cond-mat/0105592.

[22] Szabó P., Samuely P., Kacmarcik J., Klein Th., Marcus J., Fruchardt D., Miraglia S., Marcenat C. and Jansen A. G. M., cond-mat/0105598.

[23] Laube F., Goll G., Hagel J., Löhneysen H. V., Ernst D. and Wolf T., condmat/0106407.

[24] Kresin V. Z. and Parkhomenko V. P., Fiz. Tverd. Tela, 16 (1974) 3363.

[25] Manzano F. and Carrington A., cond-mat/0106166.

[26] Drechsler S.-L. and Shulga S. V., private communication. 